\title{
Inhibitory effect of liposome-entrapped lemongrass oil on the growth of Listeria monocytogenes in cheese
}

\author{
H. Y. Cui, J. Wu, and L. Lin ${ }^{1}$ \\ School of Food and Biological Engineering, Jiangsu University, Zhenjiang 212013, P. R. China
}

\begin{abstract}
Listeria monocytogenes infection in dairy products is of mounting public concern. To inhibit bacterial growth, we engineered stimuli-responsive liposomes containing lemongrass oil for this study. The controlled release of liposome-entrapped lemongrass oil is triggered by listerolysin $\mathrm{O}$, secreted by L. monocytogenes. We investigated the antibiotic activities of lemongrass oil liposomes against L. monocytogenes in cheese. We also assessed their possible effects on the quality of the cheese. Liposomes containing lemongrass oil (5.0 $\mathrm{mg} / \mathrm{mL}$ ) presented the optimal polydispersity index (0.246), zeta-potential $(-58.9 \mathrm{mV})$ and entrapment efficiency $(25.7 \%)$. The liposomes displayed satisfactory antibiotic activity against L. monocytogenes in cheese over the storage period at $4^{\circ} \mathrm{C}$. We observed no effects on the physical and sensory properties of the cheese after the liposome treatment.
\end{abstract}

Key words: listerolysin O, lemongrass oil liposome, Listeria monocytogenes, cheese

\section{INTRODUCTION}

Dairy products such as cheese play an important nutritional role in human health, because they contain a range of nutrients, including proteins, vitamins, and minerals (Songisepp et al., 2012). However, many dairy products, especially cheeses, are made from raw milk, increasing the risk of contamination by foodborne pathogens (Jamali et al., 2015). Listeria monocytogenes, one of the main pathogenic bacteria found in cheese, is remarkable because it is associated with high mortality rates (Lee et al., 2014). Furthermore, L. monocytogenes has the ability to adapt to various environments and extreme conditions, including refrigeration temperatures, water activity $(<0.94)$, and low $\mathrm{pH}(<4.4)$ (Rückerl et al., 2014). The incidence of L. monocytogenes contamination in cheese is $7.4 \%$ on average, but ranges from $1 \%$

Received March 4, 2016.

Accepted April 21, 2016.

${ }^{1}$ Corresponding author: linl@ujs.edu.cn to $22 \%$ (Guenther and Loessner, 2011). For this reason, L. monocytogenes is an important challenge for cheese production.

A number of artificial additives have been applied during cheese production to minimize the risk of $L$. monocytogenes contamination, but concerns have been raised about their potential toxicity and cancer-causing effects (Tepe et al., 2005). In that context, it is desirable to develop and use natural additives with a good safety profile and excellent antibiotic activity. Essential oils obtained from aromatic plants are of great interest because of their outstanding antibacterial and antioxidative properties (Ahmad and Viljoen, 2015).

Lemongrass (Cymbopogon citratus) has seen widespread use in folk medicine as a treatment for many disorders and infectious diseases (Balakrishnan et al., 2014). Lemongrass oil (LO) has been reported to possess excellent antibiotic activity against a considerable number of microorganisms, including bacteria, yeast, and fungi (Azarakhsh et al., 2014). However, high volatility, photosensitivity and low solubility have limited the use of essential oils in food preservation (Kim et al., 2014). Incorporating essential oils into liposome systems could reduce these effects and prolong valid antibacterial time.

Liposomes are made up of one or more concentric phospholipidic bilayers and an internal aqueous phase (Sebaaly et al., 2015). Because of their nontoxic, biocompatible, and nonimmunogenic properties, liposomes are widely used to encapsulate volatile and lipophilic ingredients such as vitamin $\mathrm{E}$ and essential oils (Toniazzo et al., 2014). Recently, a new generation of stimuliresponsive liposomes have been developed. They are focused on the intelligent release of encapsulated bioactive molecules, triggered by various stimulating agents (Kenneth et al., 2007). One of these stimulating agents is pore-forming toxin (Mueller et al., 2009; Lin et al., 2015).

Listerolysin $\mathrm{O}$ is a cholesterol-dependent pore-forming toxin secreted by many gram-positive bacteria, but L. monocytogenes is the only foodborne pathogen that secretes listerolysin O inside cells (Hamon et al., 2006). This toxin can form large pores on the phospholipidic 
bilayers of liposomes and trigger the release of antibiotic substances encapsulated in the liposomes, inactivating L. monocytogenes (Schuerch et al., 2005).

This controlled-release mechanism, triggered by pore-forming toxins, carries far-reaching implications for future food preservation, because food decay and potential foodborne diseases are caused mainly by pathogens. The purpose of this study was to investigate the antibiotic performance of liposome-entrapped LO against L. monocytogenes in cheese and the potential effects of LO on the physical and sensory attributes of the cheese itself.

\section{MATERIALS AND METHODS}

\section{Materials and Culture}

Lemongrass oil was purchased from J.E. International (Caussols, France). Fresh hard cheese (Kerrygold Cheddar) was bought from a local supermarket. Soy lecithin, cholesterol, chloroform, and polyvinylpyrrolidone were obtained from Sinopharm Chemical Reagent Co., Ltd. (Shanghai, P. R. China).

Listeria monocytogenes ATCC 19115 (China General Microbiological Culture Collection Center, Beijing, P. R. China) was stored in liquid paraffin wax at $4^{\circ} \mathrm{C}$ and cultured with shaking at $37^{\circ} \mathrm{C}$ for $48 \mathrm{~h}$.

\section{LO Encapsulation}

Lemongrass oil was entrapped in a liposome system using the method described by Cui et al. (2016) with some modifications. Initially, LO $(0,3,4,5$, and $6 \mathrm{mg} /$ $\mathrm{mL})$, soy lecithin $(20 \mathrm{mg} / \mathrm{mL})$, and cholesterol $(4 \mathrm{mg} /$ $\mathrm{mL}$ ) were mixed in chloroform, and the solvent was removed by rotary evaporation at $35-40^{\circ} \mathrm{C}$. The obtained thin film was placed in a vacuum oven at $60^{\circ} \mathrm{C}$ overnight. Then, polyvinylpyrrolidone $(1 \mathrm{mg} / \mathrm{mL})$ was mixed and hydrated in PBS (0.03 $M, \mathrm{pH} 7.2)$. The hydration solution was homogenized by an ultrasonicator (Ymnl-1000Y, Nanjing Immanuel Instrument Equipment Co. Ltd., Nanjing, P. R. China) for 35 min. After that, the LO liposome system was centrifuged for 10 $\min$ at $2,624 \times g$. Finally, the LO liposomes were filtered using a $0.22-\mu \mathrm{m}$ filter membrane.

\section{Characterization of LO Liposomes}

We characterized LO liposomes in terms of particle size, polydispersity index (PDI), surface zeta-potential, and entrapment efficiency (EE). We used a dynamic light scattering zetasizer (Nano ZS90, Malvern Instruments, Worcester, UK) to determine particle size, PDI, and surface zeta-potential. We measured EE as described in a previous study (Sebaaly et al., 2015). We analyzed different concentrations of LO $(0.2 \mathrm{mg} /$ $\mathrm{mL}, 0.4 \mathrm{mg} / \mathrm{mL}, 0.8 \mathrm{mg} / \mathrm{mL}, 1.6 \mathrm{mg} / \mathrm{mL}$, and 3.2 $\mathrm{mg} / \mathrm{mL}$ ) using GC-MS (Agilent 6890N/5973N, Agilent Technologies, Santa Clara, CA). We obtained the peak areas of geranial and neral (the main chemical components of LO) and constructed a standard curve for geranial (peak area versus concentration). The LO liposomes were dissolved in ethanol and the compounds were detected by GC-MS. All experiments were carried out 3 times.

We calculated EE using the following formula:

$$
\mathrm{EE}(\%)=\frac{A}{B} \times 100,
$$

where $A=$ concentration of encapsulated LO $(\mathrm{mg} / \mathrm{mL})$; $B=$ concentration of free LO added before encapsulation $(\mathrm{mg} / \mathrm{mL})$.

\section{Antibiotic Activity of LO Liposomes Against L. monocytogenes In Vitro}

We carried out a kill-time analysis to estimate the antibiotic activity of LO liposomes. We dissolved LO liposomes in PBS to concentrations of $2.5 \%$ ( $\mathrm{vol} / \mathrm{vol}$ ), $5.0 \%$, and $10 \%$. The PBS samples containing empty liposomes $(5 \%)$ and free LO $(0.01 \%)$ were used as controls. Next, we added cell suspensions of L. monocytogenes to the LO liposome/PBS solutions and controls. The samples had an initial bacterial concentration of $10^{5} \mathrm{cfu} / \mathrm{mL}$ and were confected and cultured at $37^{\circ} \mathrm{C}$. The number of residual bacteria were counted at 0,24 , 48, and $72 \mathrm{~h}$. All experiments were carried out 3 times via the method described by Cui et al. (2015).

Four kinds of samples were prepared, including LO (0.01\%), LO liposomes (2.5\%), PBS and LO liposomes (2.5\%) incubated with L. momocytogenes for $48 \mathrm{~h}$. Then, they were analyzed by an Agilent $7890 \mathrm{~A}$ gas chromatograph (Agilent Technologies) with an HP-5 capillary column attached. The amount of the injection was $1 \mu \mathrm{L}$. The injector and detector temperatures were 250 and $230^{\circ} \mathrm{C}$, respectively (Ahmad and Viljoen, 2015).

\section{Antibiotic Activity of LO Liposomes Against L. monocytogenes in the Model Cheese Suspension}

Cheese $(10 \mathrm{~g})$ and water $(90 \mathrm{~g})$ were blended into a slurry and sterilized. The LO liposomes were added to the cheese suspension containing L. monocytogenes (approximately $10^{3} \mathrm{cfu} / \mathrm{mL}$ ) in different concentrations $(10,20,30$, and $40 \%)$. The samples containing empty liposomes (40\%) and free LO (0.05\%) were used as 
Table 1. Characteristics (means \pm SD) of liposomes with different lemongrass oil (LO) encapsulations

\begin{tabular}{lcccc}
\hline LO $(\mathrm{mg} / \mathrm{mL})$ & Particle size $(\mathrm{nm})$ & Polydispersity index & Zeta-potential $(\mathrm{mV})$ & $\begin{array}{c}\text { Entrapment } \\
\text { efficiency }(\%)\end{array}$ \\
\hline 0 & $63.6 \pm 1.76$ & $0.235 \pm 0.023$ & $-24.8 \pm 2.38$ & 0 \\
3 & $145.7 \pm 2.97$ & $0.304 \pm 0.031$ & $-54.3 \pm 1.55$ & $18.1 \pm 1.66$ \\
4 & $163.5 \pm 2.45$ & $0.281 \pm 0.043$ & $-55.6 \pm 0.96$ & $21.5 \pm 1.09$ \\
5 & $188.1 \pm 1.82$ & $0.246 \pm 0.016$ & $-58.9 \pm 3.40$ & $25.7 \pm 0.97$ \\
6 & $196.8 \pm 3.54$ & $0.269 \pm 0.028$ & $-53.4 \pm 1.32$ & $22.4 \pm 1.43$ \\
\hline
\end{tabular}

controls. Samples were cultured at 4 and $25^{\circ} \mathrm{C}$, and we monitored the amount of change in L. monocytogenes for 7 and $14 \mathrm{~d}$. All experiments were carried out 3 times (Cui et al., 2015).

\section{Antibiotic Activity of LO Liposomes in Cheese}

Cheese samples $(3.5 \times 3 \times 1 \mathrm{~cm})$ were inoculated with $L$. monocytogenes suspension to obtain an initial bacterial concentration of $10^{3} \mathrm{cfu} / \mathrm{g}$, and then samples were maintained for $10 \mathrm{~min}$ at room temperature under sterile conditions. The inoculated samples were sprayed with LO liposomes (40\%) to a dose of $1 \mathrm{~mL} / 100 \mathrm{~g}$, or with empty liposomes or free LO at equivalent doses. All samples were packaged in sterile bags and stored at $4^{\circ} \mathrm{C}$ for $15 \mathrm{~d}$. Inoculated cheese samples with no treatments were tested as controls. We performed microbial analysis using Listeria Chromogenic Medium (Huankai Microbial Technology Co. Ltd., Guangzhou, P. R. China) and plate colony counting. All experiments were carried out 3 times.

\section{Food Physical Quality Analysis}

To assess the possible effects of LO liposomes on cheese, we measured the physical qualities (surface color and texture) of the cheese samples. Fresh cheese samples $(3.5 \times 3 \times 1 \mathrm{~cm})$ without $L$. monocytogenes treatment were sprayed with LO liposomes to a dose of $1 \mathrm{~mL} / 100 \mathrm{~g}$. Samples without LO liposomes were used as controls. All samples were packaged in sterile bags and stored at $4^{\circ} \mathrm{C}$ for $15 \mathrm{~d}$. Surface-color analysis was carried out using a chromatic meter (Color Quest XE, HunterLab, Reston, VA) in the dark, including $\mathrm{L}^{*}$ (lightness), a* (redness), and $\mathrm{b}^{*}$ (yellowness) values. The measured aperture size of the chromatic meter was $19.0 \mathrm{~mm}$. We used a xenon lamp as an illuminant. The standard observer angle was $8^{\circ}$. The instrument was calibrated using a standard white plate before measurement.

We analyzed the texture properties (hardness, springiness, cohesiveness, gumminess, and chewiness) of the cheese samples (diameter: $3 \mathrm{~cm}$; thickness: $2 \mathrm{~cm}$ ) using a texture analyzer (TA.XT. Plus, Stable Micro Systems
Ltd., Godalming, UK). Samples were compressed twice at a speed of $2.00 \mathrm{~mm} / \mathrm{s}$ and a trigger force of $0.005 \mathrm{~kg}$. All experiments were carried out 3 times.

\section{Sensory Evaluation}

Twenty untrained panelists (students and staff at Jiangsu University) evaluated the sensory attributes of the cheese samples. During the sensory evaluation, untreated and LO liposome-treated samples were placed randomly on uniform white coded plates. Participants scored color, taste, texture, off-flavor, and overall acceptability using a 5 -point hedonic scale (1, dislike very

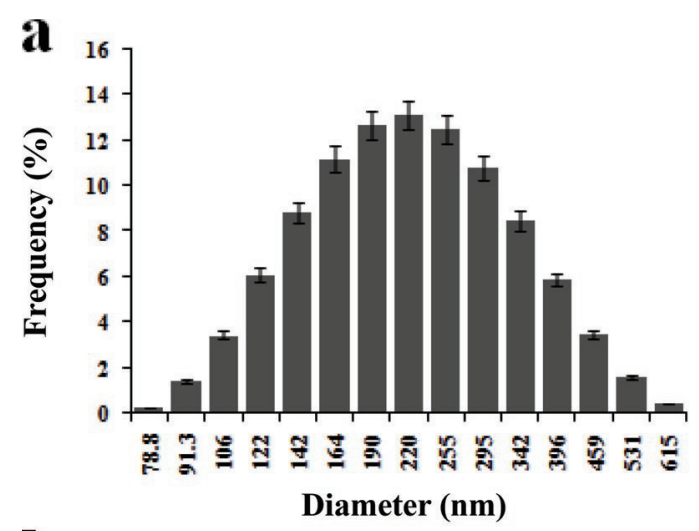

b

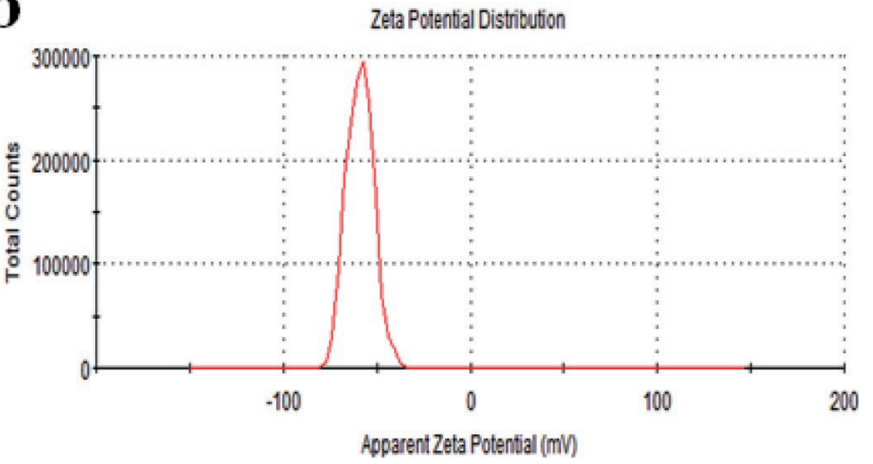

Figure 1. Size distribution (a) and surface zeta-potentials (b) of liposomes containing lemongrass oil $(5 \mathrm{mg} / \mathrm{mL})$. All experiments in this study were carried out 3 times; error bars indicate the potential error of the data. Color version available online. 
much, to 5, like very much; Ulpathakumbura et al., 2016).

\section{Statistical Analysis}

We used SPSS software (version 22.0; IBM Corp., Armonk, NY) to analyze the results. Data were reported as mean $\pm \mathrm{SD}$.

\section{RESULTS AND DISCUSSION}

\section{Characterization of LO Liposomes}

The characteristics of the LO liposomes are displayed in Table 1. Initially, the mean particle size ranged from $145.7 \pm 2.97 \mathrm{~nm}$ to $196.8 \pm 3.54 \mathrm{~nm}$. Compared with empty liposomes $(63.6 \pm 1.76 \mathrm{~nm})$, the diameter of liposomes was higher after encapsulating LO. Size is a critical indicator for liposomes. Small particles (diameter $<200 \mathrm{~nm}$ ) have the desired physicochemical properties and bioavailability for stable performance that leads to the sustained release of encapsulated active substances (Wang et al., 2010). Our results revealed that LO liposomes had a suitable size range and could maintain stability.

The PDI values for empty liposomes ranged from $0.235 \pm 0.023$ to $0.304 \pm 0.031$, indicating a relatively narrow distribution of particle size.

Surface zeta-potential values for LO liposomes in redistilled water ranged from $-53.4 \pm 1.32 \mathrm{mV}$ to -58.9 $\pm 3.40 \mathrm{mV}$, and for empty liposomes was $-24.8 \pm 2.38$ $\mathrm{mV}$. Conventionally, zeta-potential values of less than $-30 \mathrm{mV}$ and more than $+30 \mathrm{mV}$ are considered to indicate good stability, avoiding aggregation or precipitation (Lu et al., 2014; Natrajan et al., 2015). Therefore, the LO liposome suspension could be a stable system compared with empty liposomes.

The EE of LO liposomes was analyzed using GCMS. The linear equation of LO standard curve was as follows: $\mathrm{y}=33,730,030.3427 \mathrm{x}+801,175.3750\left(\mathrm{R}^{2}=\right.$ 0.9995). According to the linear equation, the $\mathrm{EE}$ of $\mathrm{LO}$ in the liposome system ranged from $18.1 \pm 1.66 \%$ to $25.7 \pm 0.97 \%$ (Table 1). The liposome EE peaked at a $5 \mathrm{mg} / \mathrm{mL}$ of LO, declining slightly at a concentration of $6 \mathrm{mg} / \mathrm{mL}$. However, EE values for LO liposomes were low. During measurement of EE, a small proportion of a

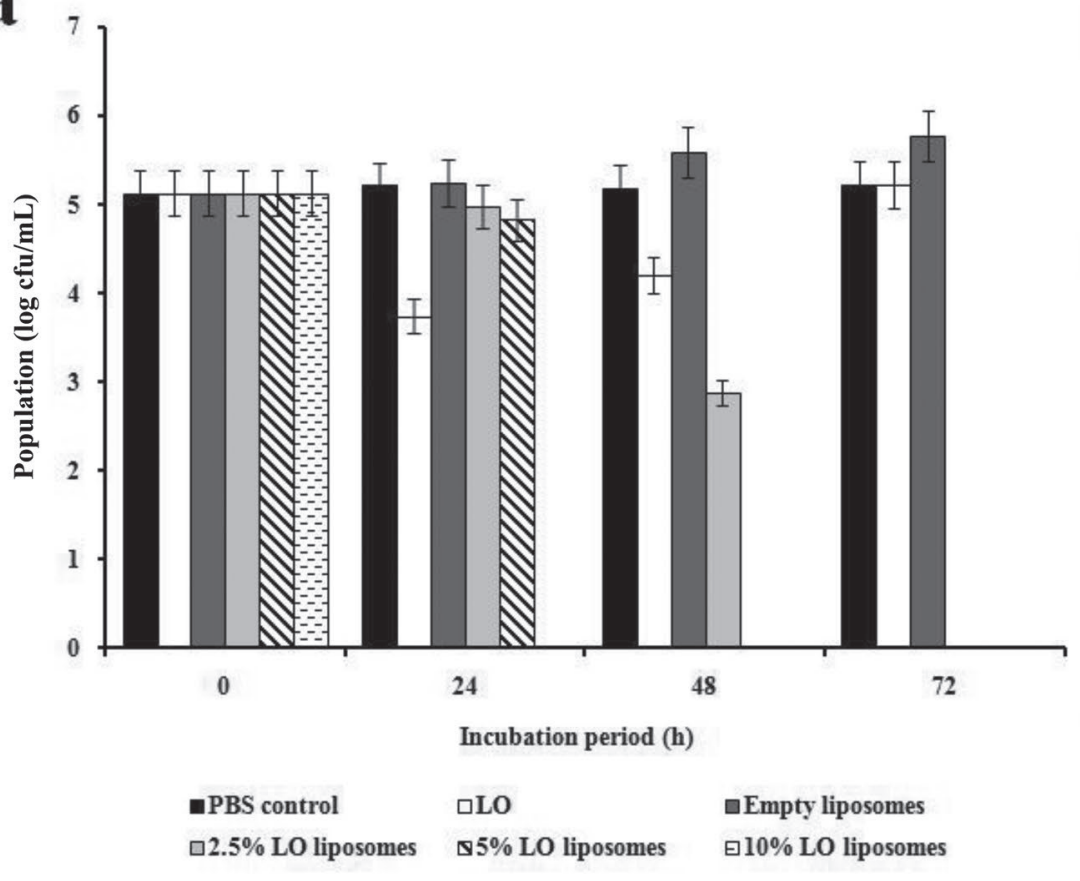

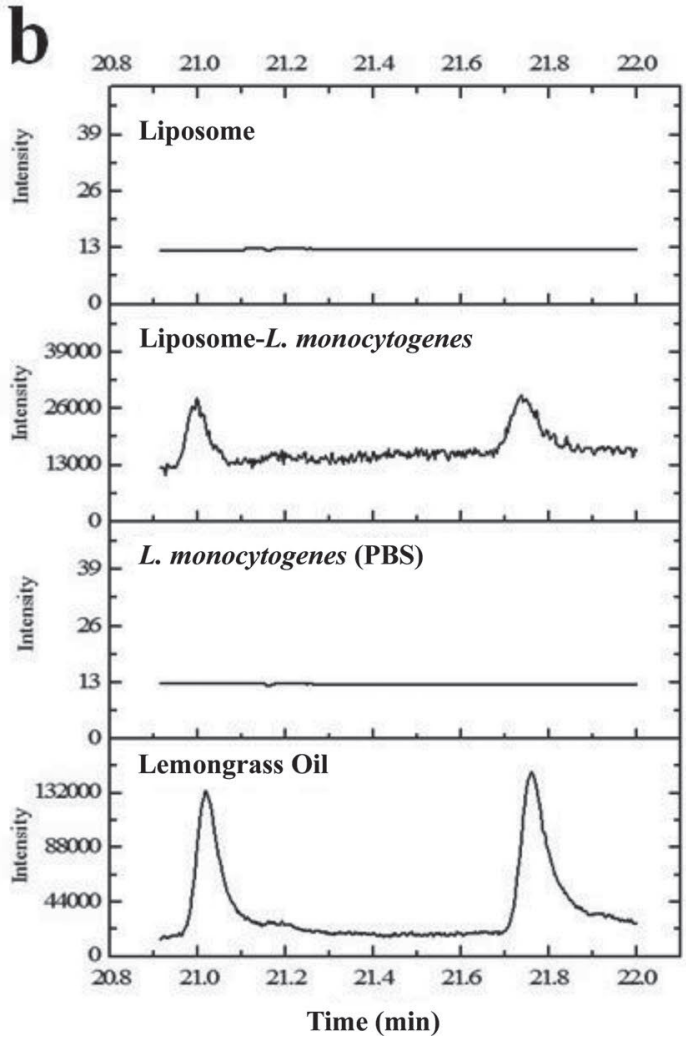

Figure 2. (a) Kill-time analysis of lemongrass oil (LO) liposomes for Listeria monocytogenes; (b) gas chromatogram of LO (0.01\%), LO liposomes (2.5\%), PBS and LO liposomes (2.5\%) incubated with L. monocytogenes. All experiments in this study were carried out 3 times; error bars indicate the potential error of the data. 
the LO liposomes could have remained in the supernatant after centrifugation, resulting in loss of LO.

Based on the above analysis, we chose LO at a concentration of $5 \mathrm{mg} / \mathrm{mL}$ to engineer liposomes for further study because this concentration had the desired PDI, zeta-potential, and EE. The particle size distribution and zeta-potential of the LO liposomes $(5 \mathrm{mg} / \mathrm{mL})$ are displayed in Figure 1.

\section{Antibiotic Activity of LO Liposomes Against L. monocytogenes In Vitro}

Figure 2a displays the results of the kill-time analysis of LO liposomes on L. monocytogenes in PBS. The num- ber of $L$. monocytogenes in empty liposomes samples increased slightly during incubation compared with controls. We found that free LO $(0.01 \%)$ inhibited the growth of L. monocytogenes after $24 \mathrm{~h}$ of incubation, but that the number of $L$. monocytogenes increased after $48 \mathrm{~h}$ because free LO was highly volatile and was lost over the incubation period. We also observed that LO liposomes at concentrations of $10 \%$ significantly inhibited the growth of L. monocytogenes after $24 \mathrm{~h}$ of incubation. Finally, we found $99.4 \%$ and $99.999 \%$ reductions in population at concentrations of 2.5 and $5.0 \%$ after $48 \mathrm{~h}$ of incubation, respectively. The results indicated that LO liposomes could achieve the desired inhibitory effect against L. monocytogenes in vitro.
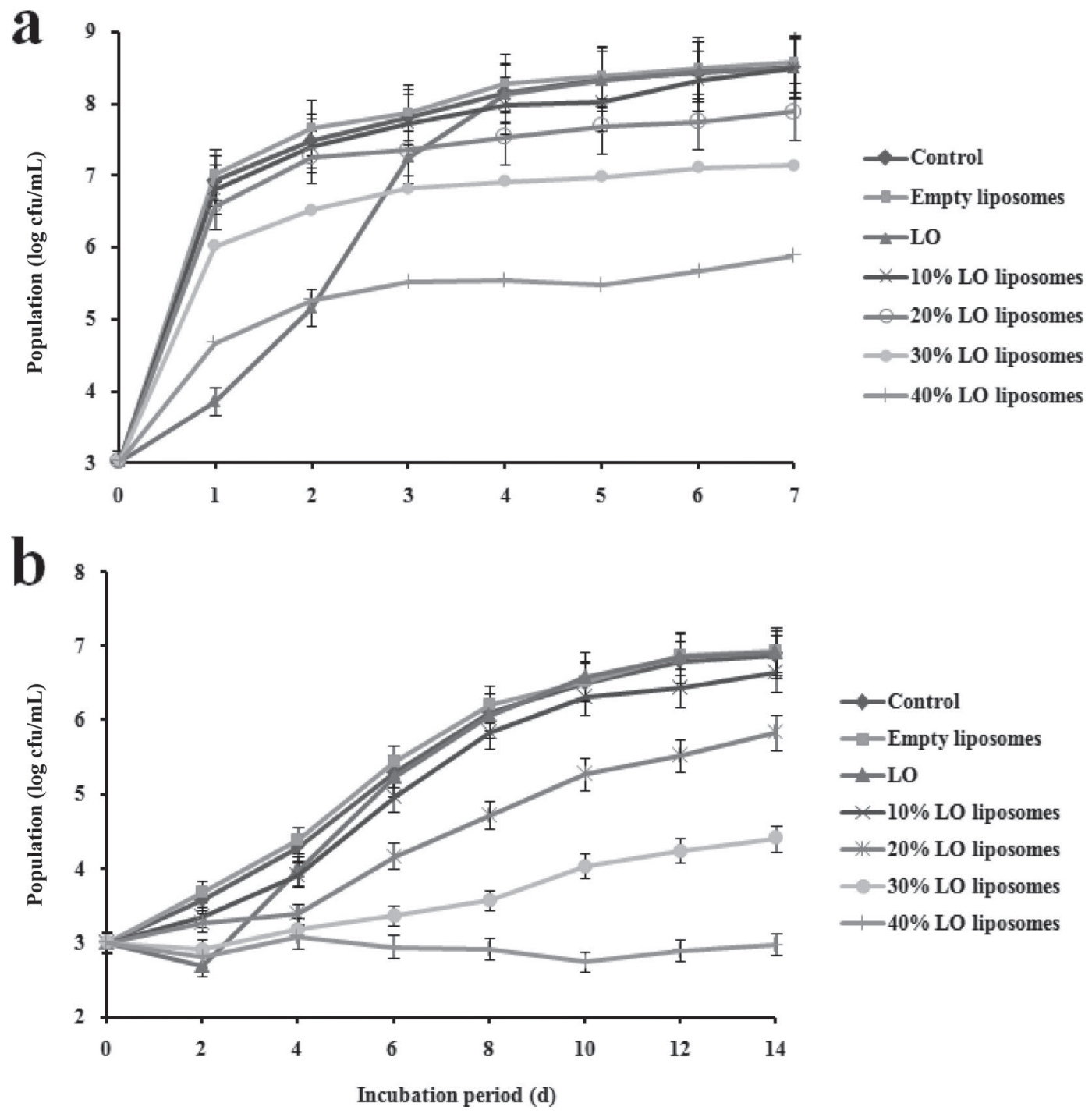

Figure 3. Antibacterial activity of lemongrass oil (LO) liposomes [PBS control, empty liposomes (40\%), LO (0.05\%), LO liposomes (10, 20, $30,40 \%)$ against Listeria monocytogenes in the model cheese suspension at (a) $25^{\circ} \mathrm{C}$, and (b) $4^{\circ} \mathrm{C}$. All experiments in this study were carried out 3 times; error bars indicate the potential error of the data. 
Table 2. Antibiotic activity (log cfu/g; means $\pm \mathrm{SD}$ ) of lemongrass oil (LO) liposomes against Listeria monocytogenes in cheese during storage at $4^{\circ} \mathrm{C}$

\begin{tabular}{|c|c|c|c|c|c|c|}
\hline \multirow[b]{2}{*}{ Treatment } & \multicolumn{6}{|c|}{ Storage period (d) } \\
\hline & 0 & 3 & 6 & 9 & 12 & 15 \\
\hline Empty liposomes & $3.02 \pm 0.57$ & $3.67 \pm 0.26$ & $4.42 \pm 0.48$ & $5.53 \pm 0.34$ & $6.23 \pm 0.52$ & $6.63 \pm 0.29$ \\
\hline $\mathrm{LO}$ & $3.02 \pm 0.57$ & $2.99 \pm 0.36$ & $3.96 \pm 0.43$ & $5.17 \pm 0.21$ & $6.16 \pm 0.37$ & $6.64 \pm 0.45$ \\
\hline LO liposomes & $3.02 \pm 0.31$ & $2.89 \pm 0.12$ & $3.01 \pm 0.41$ & $2.96 \pm 0.17$ & $2.86 \pm 0.32$ & $2.78 \pm 0.39$ \\
\hline
\end{tabular}

Results from the partial gas chromatography are shown in Figure 2b. We found no characteristic peaks of geranial and neral in the samples of PBS and LO liposomes $(2.5 \%)$ solution without L. monocytogenes, showing that LO had practically no leakage from the liposome system. However, we did observe the 2 characteristic peaks in the liposomes incubated with L. monocytogenes, demonstrating that the prepared liposomes could intelligently release the antibacterial ingredient and effectively inhibit the growth of the bacteria. The results also indicated that the release of LO liposomes could be triggered by listerolysin $\mathrm{O}$ and lead to the leakage of LO, consistent with our hypothesis.

\section{Antibiotic Activity of LO Liposomes Against L. monocytogenes in the Model Cheese Suspension}

Results for the suppressive effects of LO liposomes on $L$. monocytogenes in the model cheese suspension at $25^{\circ} \mathrm{C}$ and $4^{\circ} \mathrm{C}$ are displayed in Figure 3 . The number of L. monocytogenes generally increased with incubation time. The number of $L$. monocytogenes in the empty liposomes samples were slightly higher than in controls during the incubation period. The number of L. monocytogenes in the LO $(0.05 \%)$ samples increased slowly on the first and second days, and then rapidly after 48 $\mathrm{h}$ because of the high volatility of LO. We also observed a positive correlation between the the concentration of LO liposomes and the inhibitory effect on $L$. monocytogenes during the incubation period. After $1 \mathrm{~d}$ of incubation at $25^{\circ} \mathrm{C}$, the numbers of bacteria treated by the liposomes $(10,20$, and $30 \%)$ were increased by 3.78 ,
3.56 , and $3.00 \log _{10}$ steps respectively, but those treated by the liposomes (40\%) were increased by only 1.65 $\log _{10}$ steps. Furthermore, the liposomes $(40 \%)$ showed distinctly antibacterial activity against L. monocytogenes throughout the incubation period compared with other concentrations. Similar results were also demonstrated in the cheese suspension incubated at $4^{\circ} \mathrm{C}$. The LO liposomes at high concentration demonstrated antilisterial activity as a result of the proteins and fat in the cheese suspension; those components could affect the antibiotic activities of the liposomes (Liu and Yang, 2012). Therefore, we chose LO liposomes (40\%) for further antimicrobial and quality analyses.

\section{Antibiotic Activity of LO Liposomes in Cheese}

Table 2 displays the antibiotic activity of LO liposomes against $L$. monocytogenes in cheese during storage at $4^{\circ} \mathrm{C}$. Generally, L. monocytogenes growth in the cheese blocks was slower than in the cheese suspension at the same temperature, possibly because of lower moisture in the cheese blocks. The numbers of L. monocytogenes in the control samples increased steadily over the storage period and reached $6.58 \mathrm{log} \mathrm{cfu} / \mathrm{g}$ at $15 \mathrm{~d}$. The number of $L$. monocytogenes in empty liposome samples was slightly higher than control samples during the incubation period. The number of $L$. monocytogenes in LO $(5 \%)$ samples increased slowly on the third day, but rapidly after $3 \mathrm{~d}$ because of the high volatility of LO. However, bacteria counts were markedly inhibited by LO liposomes (40\%). The results indicated that LO liposomes showed positive inhibitory activity against $L$.

Table 3. Changes in surface-color values (means \pm SD) of cheese samples with or without lemongrass oil liposome treatment during storage at $4^{\circ} \mathrm{C}$

\begin{tabular}{lrrrrr}
\hline & \multicolumn{2}{c}{ Fresh } & & \multicolumn{2}{c}{$15 \mathrm{~d}$} \\
\cline { 2 - 3 } \cline { 5 - 6 } Color properties & \multicolumn{1}{c}{ Control } & Liposomes & & Control & Liposomes \\
\hline $\mathrm{L}^{*}$ & $72.457 \pm 0.012$ & $71.523 \pm 0.033$ & & $70.233 \pm 0.043$ & $70.102 \pm 0.077$ \\
$\mathrm{a}^{*}$ & $0.847 \pm 0.017$ & $0.896 \pm 0.025$ & & $0.824 \pm 0.029$ & $0.811 \pm 0.054$ \\
$\mathrm{~b}^{*}$ & $24.573 \pm 0.069$ & $25.147 \pm 0.019$ & & $25.846 \pm 0.031$ & $25.598 \pm 0.067$ \\
\hline
\end{tabular}


Table 4. Texture analysis (means $\pm \mathrm{SD}$ ) of cheese samples with or without lemongrass oil liposome treatment during storage at $4^{\circ} \mathrm{C}$

\begin{tabular}{lccccc}
\hline & \multicolumn{2}{c}{ Fresh } & & \multicolumn{2}{c}{$15 \mathrm{~d}$} \\
\cline { 2 - 3 } \cline { 5 - 6 } Texture parameters & Control & Liposomes & & Control & Liposomes \\
\hline Hardness (kg) & $22.61 \pm 1.06$ & $21.08 \pm 1.32$ & & $24.13 \pm 0.93$ & $23.84 \pm 1.15$ \\
Cohesion (\%) & $0.567 \pm 0.001$ & $0.559 \pm 0.001$ & & $0.586 \pm 0.002$ & $0.599 \pm 0.001$ \\
Springiness (mm) & $0.83 \pm 0.39$ & $0.86 \pm 0.24$ & & $0.90 \pm 0.27$ & $0.92 \pm 0.33$ \\
Gumminess (kg) & $23.29 \pm 0.14$ & $22.97 \pm 0.22$ & & $24.66 \pm 0.16$ & $24.41 \pm 0.19$ \\
Chewiness (kg) & $12.45 \pm 0.56$ & $12.33 \pm 0.77$ & & $13.35 \pm 0.45$ & $13.29 \pm 0.61$ \\
\hline
\end{tabular}

monocytogenes in cheese blocks, and that LO liposomes could be a promising antiseptic for cheese preservation.

\section{Food Physical Quality Analysis}

The surface-color values of the cheese samples with or without LO liposomes during the storage period are displayed in Table 3. Generally, $\mathrm{L}^{*}$ and $\mathrm{a}^{*}$ values decreased and the $b^{*}$ values increased. The $L^{*}, a^{*}$, and $b^{*}$ values of cheese samples treated with the liposomes fluctuated slightly compared with controls, but we observed no significant differences between them over the same storage time. The results demonstrated that the surface color of the samples was not affected by the liposome treatment.

The results of the texture parameter analysis are presented in Table 4. During the storage period, the texture property values increased slightly for all samples; this may have been caused by lower moisture content during storage time (Youssef et al., 2015). However, we observed no significant differences in textural parameters between the control and liposome-treated samples over same storage time. The results showed that the cheese texture was barely altered by LO liposome treatment.

\section{Sensory Evaluation}

Sensory properties such as aroma and taste are crucial features of cheese, affecting consumers' purchas- ing decisions. The results of the sensory evaluation are shown in Table 5. The sensory score of the cheese samples declined slightly over the storage period, but we observed no significant differences between the control and LO liposome-treated samples. Generally, the sensory quality of the cheese samples was maintained with LO liposome treatment.

\section{CONCLUSIONS}

This work investigated the antilisterial activity of liposomes containing LO on cheese. Desired antibiotic activity against L. monocytogenes was achieved after treatment with LO liposomes without affecting the quality of the cheese samples. Correspondingly, the shelf life of the cheese samples was extended markedly. Liposomal encapsulation technology enriches the possible application of essential oils in the dairy industry. Liposome-entrapped essential oils may have a place in the fight against foodborne pathogens.

\section{ACKNOWLEDGMENTS}

The authors appreciate the financial support provided by the National Natural Science Foundation of China (Beijing, China; 31301573), the Natural Science Foundation of Jiangsu Province (Nanjing, China;

Table 5. Sensory evaluation of cheese samples with or without lemongrass oil liposome treatment during storage at $4^{\circ} \mathrm{C}$

\begin{tabular}{|c|c|c|c|c|}
\hline \multirow[b]{2}{*}{ Sensory parameter ${ }^{1}$} & \multicolumn{2}{|c|}{ Fresh } & \multicolumn{2}{|c|}{$15 \mathrm{~d}$} \\
\hline & Control & Liposomes & Control & Liposomes \\
\hline Color & $2.87 \pm 0.43$ & $2.84 \pm 0.22$ & $2.67 \pm 0.61$ & $2.63 \pm 0.37$ \\
\hline Taste & $2.56 \pm 0.38$ & $2.53 \pm 0.16$ & $2.47 \pm 0.54$ & $2.42 \pm 0.49$ \\
\hline Texture & $2.66 \pm 0.51$ & $2.61 \pm 0.64$ & $2.49 \pm 0.28$ & $2.45 \pm 0.43$ \\
\hline Off-flavor & $2.81 \pm 0.19$ & $2.78 \pm 0.23$ & $2.72 \pm 0.43$ & $2.67 \pm 0.51$ \\
\hline Overall acceptability & $2.72 \pm 0.35$ & $2.77 \pm 0.27$ & $2.63 \pm 0.22$ & $2.59 \pm 0.46$ \\
\hline
\end{tabular}

${ }^{1}$ Data are the means $( \pm \mathrm{SD})$ of a 5 -point hedonic scale: $1=$ dislike very much; $2=$ dislike somewhat; $3=$ neither like nor dislike; $4=$ like somewhat; $5=$ like very much. 
BK20130493), and the Jiangsu University Research Foundation (Zhenjiang, China; 08JDG039, 11JDG050).

\section{REFERENCES}

Ahmad, A., and A. Viljoen. 2015. The in vitro antimicrobial activity of Cymbopogon essential oil (lemon grass) and its interaction with silver ions. Phytomedicine 22:657-665.

Azarakhsh, N., A. Osman, H. M. Ghazali, C. P. Tan, and N. M. Adzahan. 2014. Lemongrass essential oil incorporated into alginatebased edible coating for shelf-life extension and quality retention of fresh-cut pineapple. Postharvest Biol. Technol. 88:1-7.

Balakrishnan, B. S. Paramasivam, and A. Arulkumar. 2014. Evaluation of the lemongrass plant (Cymbopogon citratus) extracted in different solvents for antioxidant and antibacterial activity against human pathogens. Asian Pac. J. Trop. Dis. 4(Suppl. 1):S134-S139.

Cui, H. Y., C. T. Zhao, and L. Lin. 2015. The specific antibacterial activity of liposome-encapsulated Clove oil and its application in tofu. Food Contr. 56:128-134.

Cui, H. Y., H. Zhou, and L. Lin. 2016. The specific antibacterial effect of the Salvia oil nanoliposomes against Staphylococcus aureus biofilms on milk container. Food Contr. 61:92-98.

Guenther, S., and M. J. Loessner. 2011. Bacteriophage biocontrol of Listeria monocytogenes on soft ripened white mold and red-smear cheeses. Bacteriophage 1:94-100.

Hamon, M., H. Bierne, and P. Cossart. 2006. Listeria monocytogenes: A multifaceted model. Nat. Rev. Microbiol. 4:423-434.

Jamali, H., M. Paydar, B. Radmehr, S. Ismail, and A. Dadrasnia 2015. Prevalence and antimicrobial resistance of Staphylococcus aureus isolated from raw milk and dairy products. Food Contr. 54:383-388.

Howard, K. A., M. Dong, D. Oupicky, H. S. Bisht, C. Buss, F. Besenbacher, and J. Kjems. 2007. Nanocarrier stimuli-activated gene delivery. Small 3:54-57.

Kim, I. H., Y. A. Oh, H. Lee, K. B. Song, and S. C. Min. 2014. Grape berry coatings of lemongrass oil-incorporating nanoemulsion. Food Sci. Technol. (Campinas.) 58:1-10.

Lee, J. Y., B. S. Jung, H. J. Yoon, K. T. Kim, H. D. Paik, and J. Lee. 2014. Predictive model for the growth kinetics of Listeria monocytogenes in raw pork meat as a function of temperature. Food Contr. 44:16-21.

Lin, L., H. Y. Cui, H. Zhou, X. J. Zhang, L. Liu, C. Bortolini, M. L. Chen, and M. D. Dong. 2015. Nanoliposomes containing eucalyptus citriodora antibiotics for specific antimicrobial activity. Chem. Commun. (Camb.) 51:2653-2655.

Liu, T. T., and T. S. Yang. 2012. Antimicrobial impact of the components of essential oil of Litsea cubeba from Taiwan and antimi- crobial activity of the oil in food systems. Int. J. Food Microbiol. 156:68-75.

Lu, Q., P. M. Lu, J. H. Piao, X. L. Xu, J. Chen, L. Zhu, and J. G. Jiang. 2014. Preparation and physicochemical characteristics of an allicin nanoliposome and its release behavior. Food Sci. Technol. (Campinas.) 57:686-695.

Mueller, M., U. Grauschopf, T. Maier, R. Glockshuber, and N. Ban. 2009. The structure of a cytolytic alpha-helical toxin pore reveals its assembly mechanism. Nature 459:726-730.

Natrajan, D., S. Srinivasan, K. Sundar, and A. Ravindran. 2015. Formulation of essential oil-loaded chitosan-alginate nanocapsules. J. Food Drug Anal. 23:560-568.

Rückerl, I., M. Muhterem-Uyar, S. Muri-Klinger, K. H. Wagner, M. Wagner, and B. Stessl. 2014. L. monocytogenes in a cheese processing facility: Learning from contamination scenarios over three years of sampling. Int. J. Food Microbiol. 189:98-105.

Schuerch, D. W., E. M. Wilson-Kubalek, and R. K. Tweten. 2005. Molecular basis of listeriolysin $\mathrm{O}$ pH dependence. Proc. Natl. Acad. Sci. USA 102:12537-12542.

Sebaaly, C. A. Jraij, H. Fessi, C. Charcosset, and H. Greige-Gerges. 2015. Preparation and characterization of clove essential oil-loaded liposomes. Food Chem. 178:52-62.

Songisepp, E., P. Hütt, M. Rätsep, E. Shkut, S. Kõljalg, K. Truusalu, J. Stsepetova, I. Smidt, H. Kolk, M. Zagura, and M. Mikelsaar. 2012. Safety of a probiotic cheese containing Lactobacillus plantarum Tensia according to a variety of health indices in different age groups. J. Dairy Sci. 95:5495-5509.

Tepe, B., D. Daferera, A. Sokmen, M. Sokmen, and M. Polissiou. 2005. Antimicrobial and antioxidant activities of the essential oil and various extracts of Salvia tomentosa Miller (Lamiaceae). Food Chem. 90:333-340.

Toniazzo, T., I. F. Berbel, S. Cho, C. S. Fávaro-Trindade, I. C. F Moraes, and S. C. Pinho. 2014. Beta-carotene-loaded liposome dispersions stabilized with xanthan and guar gums: Physico-chemical stability and feasibility of application in yogurt. Food Sci. Technol. (Campinas) 59:1265-1267.

Ulpathakumbura, C. P., C. S. Ranadheera, N. D. Senavirathne, L. P. I. N. P. Jayawardene, P. H. P. Prasanna, and J. K. Vidanarachchi. 2016. Effect of biopreservatives on microbial, physico-chemical and sensory properties of Cheddar cheese. Food Biosci. 13:21-25.

Wang, H., P. Zhao, X. Liang, X. Gong, T. Song, R. Niu, and J. Chang. 2010. Folate-PEG coated cationic modified chitosan-cholesterol liposomes for tumor-targeted drug delivery. Biomaterials 31:41294138

Youssef, A. M., S. M. El-Sayed, H. H. Salama, H. S. El-Sayed, and A. Dufresne. 2015. Evaluation of bionanocomposites as packaging material on properties of soft white cheese during storage period. Carbohydr. Polym. 132:274-285. 